\title{
Dislocation density and graphitization of diamond crystals
}

\author{
C. Pantea, ${ }^{1}$ J. Gubicza, ${ }^{2,3}$ T. Ungar, ${ }^{2}$ G. A. Voronin, ${ }^{1}$ and T. W. Zerda ${ }^{1}$ \\ ${ }^{1}$ Department of Physics and Astronomy, TCU, Fort Worth, Texas 76129 \\ ${ }^{2}$ Department of General Physics, Eötvös University, Budapest, H-1518, P.O. Box 32, Hungary \\ ${ }^{3}$ Department of Solid State Physics, Eötvös University, Budapest, P.O. Box 32, H-1518, Hungary
}

(Received 25 April 2002; published 16 September 2002)

\begin{abstract}
Two sets of diamond specimens compressed at $2 \mathrm{GPa}$ at temperatures varying between $1060 \mathrm{~K}$ and $1760 \mathrm{~K}$ were prepared; one in which graphitization was promoted by the presence of water and another in which graphitization of diamond was practically absent. X-ray diffraction peak profiles of both sets were analyzed for the microstructure by using the modified Williamson-Hall method and by fitting the Fourier coefficients of the measured profiles by theoretical functions for crystallite size and lattice strain. The procedures determined mean size and size distribution of crystallites as well as the density and the character of the dislocations. The same experimental conditions resulted in different microstructures for the two sets of samples. They were explained in terms of hydrostatic conditions present in the graphitized samples.
\end{abstract}

DOI: 10.1103/PhysRevB.66.094106

PACS number(s): 61.72.Dd, 61.72.Lk, 81.05.Uw, 61.50.Ks

\section{INTRODUCTION}

The mechanism of graphitization of diamond has been extensively studied and the results can be summarized as follows. The rate of diamond-to-graphite phase transition increases with increasing temperature. To slow down this process, high pressure can be applied. At sufficiently high pressures diamond is the stable form of carbon and graphitization stops, compare phase diagrams published in Ref. 1. Catalytic effects of oxygen, water, and some metals on the diamondto-graphite transformation have been discussed in Refs. 2 and 3. The effect of oxidation in the production of graphite on the surface of diamond has been explained in details in Ref. 3. It was shown that even small amounts of oxygen have a pronounced effect on the graphitization process.

In our previous studies we showed that different mechanisms control graphitization of (100) and (111) crystal faces. ${ }^{4}$ In the former case, individual atoms break away from the surface, one at a time, and these liberated atoms form graphitic layers. In the latter case, whole sheets of carbon atoms are lifted from the (111) surface and after flattening they form graphite crystals. In each case surface defects are believed to be the nuclei for graphitization.

In this study we examine the evolution of dislocation structure in diamonds during the graphitization process. Different concentrations of dislocations were obtained by compressing diamond powders at $2 \mathrm{GPa}$ at various temperatures. All results discussed in this paper refer to quenched samples, recovered after the high temperature, high pressure treatment. Two sets of diamond samples were investigated. In one set of experiments we eliminated all external factors, except oxygen, that promote diamond-to-graphite phase transformation. In the second, we accelerated graphitization by slowly releasing water into the reaction chamber. After the pressure was released and temperature reduced to room temperature we studied microstructure of diamond samples, characterized crystallite sizes and population of defects as well as the quantity of graphite produced.

\section{EXPERIMENT}

\section{A. Sample preparation}

All samples were obtained from synthetic diamond particles (General Electric Co.) of sizes 30-40 $\mu \mathrm{m}$, compacted under high-pressure high-temperature conditions. Experiments were run in a cylinder-type apparatus at a pressure of $2.0 \mathrm{GPa}$ at selected temperatures in the $1070-1760 \mathrm{~K}$ range. A long heating time of $20 \mathrm{~min}$ was chosen for two reasons. First, the phase transition of diamond to graphite reaches equilibrium after $20 \mathrm{~min}$. Second, we wanted to obtain the most uniform stress distribution in the samples. After the first 10 min of high pressure-high temperature treatment the magnitude of stresses varies widely as indicated by fluctuating x-ray bandwidths. ${ }^{5}$ The scatter in stresses is reduced with elapsed time and after $20 \mathrm{~min}$ the peak broadenings are relatively constant.

The details of the experimental setup are given in Ref. 2. To reach high pressure a piston-cylinder system manufactured by Rockland research was used. The pressure was generated by pushing the specimen placed inside a cylindrical hole in a pressure vessel with a very snug-fitting tungsten carbide piston. High temperature was achieved by passing a current through a cylindrical graphite heater located inside the pressure vessel. W3\% Re/W25\%Re thermocouple was used to measure and control the temperature inside the sample. $^{2}$

Talc tubes were used for electrical isolation and as holders for diamond powder. Two different sets of experiments were conducted. In the first series we used raw talc, which during heat treatment released water into the reaction chamber. Water acted as a catalyst for the graphitization process. In the second series of experiments we used water-free talc, which did not exhibit catalytic effects. It was obtained from raw talc by heating for $30 \mathrm{~min}$ at $1100 \mathrm{~K}$. The heat-treated talc did not produce water during subsequent heating. The samples obtained using different talc holders will be referred to as not-graphitized diamond (obtained with the heat-treated talc), and graphitized diamond (raw talc). We assumed that in both series of experiments, oxygen present inside the sample 
compartment had the same effect on the graphitization process and the differences in the amount of graphite produced was mainly caused by different concentration of another catalyst, water.

High pressure, high temperature (HPHT) experiments were conducted according to the following protocol. In the first step, at room temperature, the pressure was raised to 2 $\mathrm{GPa}$. Next, temperature was increased to the desired value at a rate of $200 \mathrm{~K} / \mathrm{min}$. The samples were kept at that temperature for $20 \mathrm{~min}$ and then the heating was stopped and the pressure released. Then, the temperature was decreased at the same rate of $200 \mathrm{~K} / \mathrm{min}$ to the room temperature, and the pressure released. Samples obtained by using the heat treated talc at the maximum temperature of $1070 \mathrm{~K}, 1270 \mathrm{~K}, 1470$ $\mathrm{K}$, and $1760 \mathrm{~K}$ are called D0, D1, D2, and D3, respectively. Samples that were allowed to partially graphitize are called DG0 (1070 K), DG1 (1270 K), DG2 (1470 K), and DG3 $(1760 \mathrm{~K})$. For sample DG0 the results were identical with those for sample D0. These two samples will be referred as D0.

\section{B. X-ray diffraction experiments}

X-ray diffraction spectra of samples recovered from high pressure and high temperature treatment were obtained on a conventional powder diffractometer (Philips X'pert) using $\mathrm{Cu} K \alpha$ radiation and pyrolitic graphite secondary monochromator. For x-ray diffraction peak profile analysis the first five peaks of diamond were measured individually by a special double-crystal high-resolution diffractometer with a very small instrumental broadening [with the sample to detector distance $\left.l=500 \mathrm{~mm} \Delta(2 \Theta)_{\text {instr }}=0.012^{\circ}\right]$ attached to a high brilliance rotating anode (Nonius, Holland). In this latter case monochromatized $\mathrm{Cu} K \alpha_{1}$ incident radiation was used.

\section{Evaluation procedure of $x$-ray diffraction profiles}

The evaluation of the peak profiles was performed by the Multiple Whole-Profile fitting (MWP) procedure. ${ }^{6,7}$ In this method the Fourier coefficients of the measured profiles were fitted by the product of the theoretical functions for size $\left(A^{s}\right)$ and strain $\left(A^{d}\right)$ peak broadening. In the calculation of the theoretical functions the crystallites were assumed to have spherical form with log-normal size distribution and the strains were assumed to be caused by dislocations. According to this model of the microstructure, the theoretical function for the size and strain Fourier coefficients are

$$
\begin{aligned}
A^{S}(L) \sim & \frac{m^{3} \exp \left(4.5 \sigma^{2}\right)}{3} \operatorname{erfc}\left[\frac{\ln (|L| / m)}{\sqrt{2} \sigma}-1.5 \sqrt{2} \sigma\right] \\
& -\frac{m^{2} \exp \left(2 \sigma^{2}\right)|L|}{2} \operatorname{erfc}\left[\frac{\ln (|L| / m)}{\sqrt{2} \sigma}-\sqrt{2} \sigma\right] \\
& +\frac{|L|^{3}}{6} \operatorname{erfc}\left[\frac{\ln (|L| / m)}{\sqrt{2} \sigma}\right],
\end{aligned}
$$

and

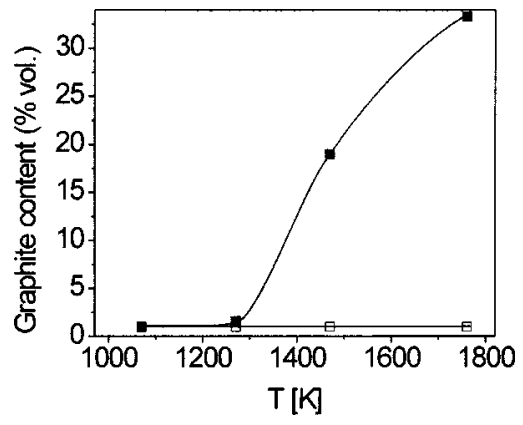

FIG. 1. Graphite content (vol \%) after compressing at $2 \mathrm{GPa}$ as a function of temperature. Solid squares: graphitized diamond; open squares: not-graphitized diamond.

$$
A^{D}(L)=\exp \left[-\rho B L^{2} f(\eta) K^{2} \bar{C}\right],
$$

respectively, where $L$ is the Fourier variable, $m$ is the median, and $\sigma$ is the variance of the log-normal size distribution function, erfc is the complementary error function, $\rho$ is the dislocation density, $B=\pi b^{2} / 2, b$ is the absolute value of the Burgers vector, $K$ is the absolute value of the diffraction vector, $\eta \sim L / R_{e}, R_{e}$ is the effective outer cutoff radius of dislocations, and $f(\eta)$ is a function derived explicitly by Wilkens [see Eqs. (A6)-(A8) in Ref. 8 and Eqs. (22) and (23) in Ref. 6]. $C$ is the dislocation contrast factor which is introduced to take into account the strain anisotropy of dislocations. For an untextured polycrystalline material or for a single crystal with equally populated dislocation slip systems, the contrast factors in Eq. (2) are the average of the individual $C$ factors either over the permutations of the indices of reflections, $h k l$ or over the dislocation population. ${ }^{9}$ This contrast factor is called the average contrast factor, $\bar{C}$. Based on the theory of peak broadening caused by dislocations, it can be shown that in an untextured cubic polycrystalline specimen the values of $\bar{C}$ are simple functions of the invariants of the fourth order polynomials of $h k l,{ }^{10}$

$$
\bar{C}=\bar{C}_{h 00}\left\{1-q\left(h^{2} k^{2}+h^{2} l^{2}+k^{2} l^{2}\right) /\left(h^{2}+k^{2}+l^{2}\right)^{2}\right\},
$$

where $\bar{C}_{h 00}$ is the average dislocation contrast factor for the $h 00$ reflections and $q$ is a parameter depending on the elastic constants of the crystal and on the character of dislocations (e.g., edge or screw type).

As a result of the MWP fitting procedure the median $(m)$ and the variance $(\sigma)$ of the size distribution, the density $(\rho)$ and the character (edge or screw) of dislocations were obtained. The volume weighted mean crystallite size was also calculated from the $m$ and $\sigma$ values as ${ }^{6}$

$$
\langle x\rangle_{\mathrm{vol}}=m \exp \left(3.5 \sigma^{2}\right) \text {. }
$$

\section{RESULTS}

The quantity of graphite obtained from diamond crystals was determined from the $\mathrm{x}$-ray powder diffraction spectra by determining relative intensity ratio of the graphite (002) and diamond (111) peaks. The graphite content (see Fig. 1) in the samples obtained using heat-treated talc was always below 


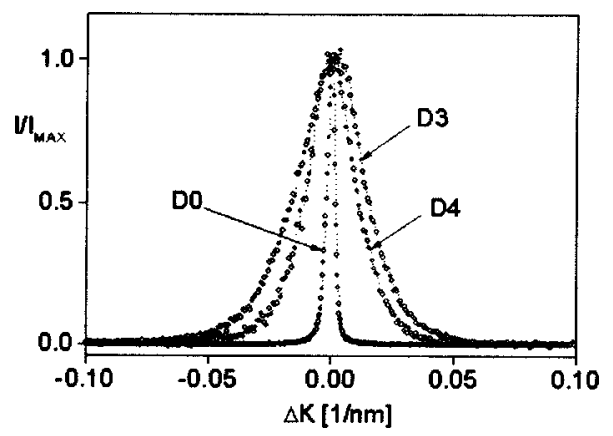

FIG. 2. (220) peak profiles of not-graphitized diamond samples.

$1 \%$. However, when raw talc was used, the content of graphite was temperature-dependent, and for the highest temperature used, it reached $33 \%$. These results are limited to the crystals with $30-40 \mu \mathrm{m}$ initial size. For crystals of other sizes, different amounts of produced graphite may be expected. $^{2}$

High-resolution x-ray measurements conducted on a Nonius rotating anode diffractometer provided information on crystal sizes and population of dislocations. These highresolution spectra were obtained for five peaks of diamond: (111), (220), (311), (400), and (133), the maximum number of peaks observable with $\mathrm{Cu}$ radiation. In Fig. 2 we show changes in the (220) peak profiles for the not-graphitized diamond. As temperature is increased, the peak width initially increases and after reaching a maximum at $1470 \mathrm{~K}$ it decreases with increased temperature. Average crystallite sizes, size distributions, dislocation structure, character, and density were evaluated by the MWP method. In this analysis the whole profiles of each peak were taken into account.

\section{A. Sample D0}

For sample D0 the (111) and (311) reflections were very narrow, the full widths at half maximum (FWHM) of these peaks were equal to the instrumental broadening of $0.012^{\circ}$. This means that the sample was composed of large crystals probably much larger than $1 \mu \mathrm{m}$ ( $1 \mu \mathrm{m}$ is approximately the upper limit of the measurable size by $\mathrm{x}$ rays). Using a very narrow beam size of about $60 \times 100 \mu \mathrm{m}$ cross section the diffracted radiation was collected from only one large crystal. Here we note that for measuring each reflection in the case of the initial-state and the D0 specimen the samples had to be oriented by turning the 3 axis goniometer in such a way as if the specimen were single crystals. This means that in these cases all the five reflections: (111), (220), (311), (400), and (133) correspond to the same crystallite. In that sense, for these specimens, the experiment is a quasisingle crystal diffraction experiment, rather than a powder diffraction experiment. In Fig. 3 the FWHM values for sample D0 are shown as a function of $K(K=2 \sin \theta / \lambda)$ in the classical Williamson-Hall plot. The points in Fig. 3 do not follow any smooth curve indicating strain anisotropy caused by dislocations. In the modified Williamson-Hall plot, taking into account the anisotropic peak broadening caused by dislocations, the FWHM is plotted as a function of $K^{2} C$. In this representation the points should follow a smooth quadratic

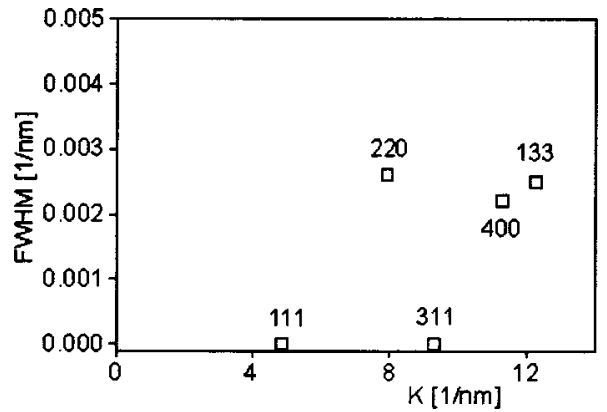

FIG. 3. The classical Williamson-Hall plot of sample D0.

curve. ${ }^{6}$ As mentioned before, in a texture free polycrystalline materials or in a single crystal where the different slip systems are randomly populated by dislocations the average contrast factors can be used. ${ }^{6}$ An attempt was made to apply the average contrast factors in the modified Williamson-Hall plot for sample D0 but the points could not be brought to follow a smooth curve for any value of the $q$ parameter [see Eq. (3)]. This means that only a specific dislocation slip system is populated and therefore individual contrast factors have to be applied in the modified Williamson-Hall plot. Analyzing the individual contrast factors corresponding to all twelve possible slip systems, it turns out that there is only one single Burgers vector, for which, the contrast factors of the (111) and (311) reflections are close to zero. Assuming that the (111) reflecting planes of the specimen are parallel to the sample surface (the specimen has been in the symmetrical diffraction position) the only dislocation satisfying this condition is of the edge-type and has the following parameters:

$$
\text { slip plane: }(1 \overline{1} \overline{1})
$$

$$
\begin{gathered}
\text { Burgers vector: } \quad \mathbf{b}=[01 \overline{1}], \\
\text { line vector: } \quad \mathbf{l}=[211]
\end{gathered}
$$

The individual contrast factors for this specimen with these diffraction and Burgers vector conditions are given in Table I. Using these values for the individual contrast factors in the modified Williamson-Hall plot the FWHM values follow a smooth quadratic curve shown in Fig. 4, justifying at the same time the applicability of this model, in accordance with the experimental conditions described in detail before. Since the FWHM of the (111) and (311) reflections are prac-

TABLE I. Dislocation contrast factors $C$ for different indices of reflections for the edge dislocations with the Burgers vector of

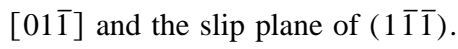

\begin{tabular}{cc}
\hline \hline$h k l$ & $C$ \\
\hline 111 & 0.0008 \\
220 & 0.0354 \\
311 & 0.0003 \\
400 & 0.0144 \\
133 & 0.0134 \\
\hline
\end{tabular}




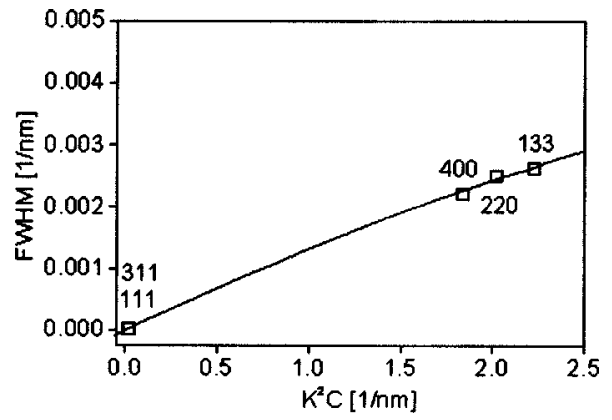

FIG. 4. The modified Williamson-Hall plot of sample D0.

tically identical to the instrumental FWHM value we conclude that the broadening of the other three reflections is only due to strain. The dislocation density has been evaluated by fitting Eq. (2) to the profile of the (220) reflection. The same procedure was applied to the initial diamond powder and the dislocation densities for both samples are given in Table II.

\section{B. Samples D1, D2, and D3}

For samples D1, D2, and D3 the concept of the average dislocation contrast factors was working well, no additional corrections were necessary. This is mainly due to the fact that the size of the crystallites has been reduced to the nanometer range. Therefore, in the present case the experiments are true powder diffraction experiments unlike in the case of the D0 specimen. The dislocation structure and crystallite size distribution were determined by the MWP fitting procedure using the average contrast factors. ${ }^{6}$ In this case an additional parameter, $q$, was obtained from the fitting procedure, which depends on the elastic constants of the crystal and the edge or screw character of dislocations. With the values for the elastic stiffness constants given in Ref. 11 and assuming the most common dislocation slip system in diamond with the Burgers vector $\mathbf{b}=a / 2\langle 110\rangle\{111\}$, the values of $q$ for pure screw and pure edge dislocations in diamond are 1.35 and 0.30 , respectively. ${ }^{12}$ The experimental value of $q$ describes the edge/screw character of dislocations. The crystallite size, the dislocation density and the $q$ parameter are summarized

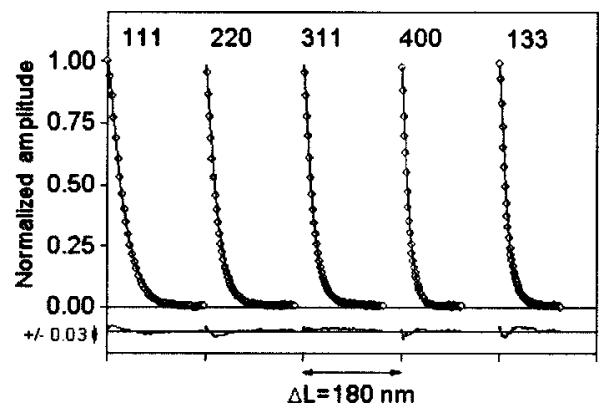

FIG. 5. Measured (open circles) and fitted (solid line) Fourier transforms of the diffraction profiles of the DG1 diamond sample. The difference between the two sets of curves is shown in the bottom part of the figure.

in Table II. It can be seen that at the lowest temperature $(1070 \mathrm{~K})$ only edge dislocations are present in the crystals. When temperature is increased to $1270 \mathrm{~K}$ the $q$ factor is 0.7 , i.e., screw dislocations appear. The $q$ factor increases with temperature and at $1470 \mathrm{~K}$ it reaches 1.2 . This means that at high temperatures the character of the dislocation structure became more screw-type. Further temperature increase did not effect the relative concentration of the screw dislocations.

The crystallite size did not change significantly with temperature, its value was about $50-80 \mathrm{~nm}$. The dislocation density first increased rapidly with temperature and reached a maximum at $1470 \mathrm{~K}$, afterwards it decreased.

\section{Samples DG1, DG2, and DG3}

Samples DG1, DG2, and DG3 contained graphite. The quantity of graphite produced at the lowest temperature, $1070 \mathrm{~K}$, was close to zero. When temperature was increased the quantity of graphite produced from diamond also increased. At the highest temperature used about $33 \%$ of diamond transformed into graphite.

Examples of measured and fitted Fourier transforms of the diffraction profiles of the DG1 diamond sample are represented in Fig. 5. The average size of the crystallites for different specimens was about 60-100 nm, see Table II. In the

TABLE II. Graphite content (volume percent) and microstructural parameters for diamond samples compressed at 2 GPa. $m$ is the median crystallite size, $\sigma$ is the size variance, $\langle x\rangle_{\text {vol }}$ is the volume-weighted mean crystallite size, $\rho$ is the density of dislocations, and $q$ is the factor describing the character of dislocations.

\begin{tabular}{|c|c|c|c|c|c|c|c|}
\hline Sample & $\begin{array}{c}T \\
(\mathrm{~K})\end{array}$ & $\begin{array}{c}\text { Graphite } \\
(\%)\end{array}$ & $\begin{array}{c}m \\
(\mathrm{~nm})\end{array}$ & $\sigma$ & $\begin{array}{c}\langle x\rangle_{\mathrm{vol}} \\
(\mathrm{nm})\end{array}$ & $\begin{array}{c}\rho \\
\left(10^{14} \mathrm{~m}^{-2}\right)\end{array}$ & $q$ \\
\hline initial & normal & & & & & & \\
\hline diamond & conditions & 0.0 & $\cdots$ & $\cdots$ & $\cdots$ & 0.3 & $\ldots$ \\
\hline D0 & 1070 & $<1.0$ & $\cdots$ & $\cdots$ & $>1000$ & 2.0 & $\cdots$ \\
\hline D1 & 1270 & $<1.0$ & 43 & 0.35 & 66 & 8.5 & 0.7 \\
\hline D2 & 1470 & $<1.0$ & 37 & 0.25 & 46 & 12.0 & 1.2 \\
\hline D3 & 1760 & $<1.0$ & 58 & 0.27 & 75 & 7.0 & 1.1 \\
\hline DG1 & 1270 & 1.6 & 52 & 0.44 & 102 & 8.5 & 0.8 \\
\hline DG2 & 1470 & 19.0 & 60 & 0.40 & 105 & 5.0 & 0.6 \\
\hline DG3 & 1760 & 33.3 & 42 & 0.35 & 64 & 6.0 & 0.6 \\
\hline
\end{tabular}




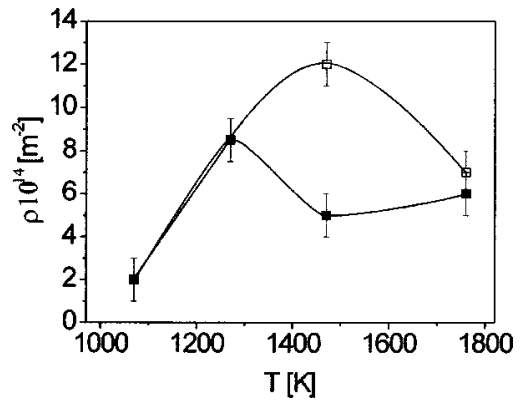

FIG. 6. Dislocation density in graphitized (solid squares) and not-graphitized (open squares) samples. Solid lines are shown to guide the eye.

MWP procedure the average dislocation contrast factors were used. The calculated dislocation density for the sample after treatment at $1270 \mathrm{~K}$ (DG1) was the same as for the not-graphitized samples, but for the sample heat treated at $1470 \mathrm{~K}$ it was about half the value obtained for the notgraphitized diamonds obtained at the same conditions. The character of dislocations in this case was changing from edge type to mixed type, and above $1270 \mathrm{~K}$ remained constant, with the $q$ factor value of $0.6-0.7$.

\section{DISCUSSION}

As expected, increased temperature and compression of diamond powder resulted in changes in crystallite sizes and the population of dislocations. Different results were obtained for samples that avoided graphitization and for those that were partially graphitized.

Raw diamond crystals had a significant concentration of dislocations which at high pressure conditions increased rapidly with increased temperature. The density of dislocations was always greater in samples that were compressed and heated in water free environments (did not graphitize) than in partially graphitized samples. At the lowest temperatures used in our experiments the difference in the population of dislocations between the sets of samples was very small, but quickly increased and reached maximum at $1470 \mathrm{~K}$ and then decreased with further increase in temperature, compare Fig. 6 . The average density of dislocation does not characterize the distribution of dislocations within the crystals. However, it is safe to assume that this distribution is not uniform, and largest concentrations of defects are expected in close proximity of contact points where two crystals press one into another. The smallest concentration is expected near voids between diamond crystals. Mobility of dislocations increases with increased temperature due to thermal activation. Dislocations may move into the interior of the crystal, consequently they do not impede the formation of new dislocations at the contact points. As a result, the dislocation density increases with temperature. As indicated by the increase of the parameter $q$, screw dislocations with low energy are preferentially produced at high pressure-high temperature conditions. At very high temperatures $(1760 \mathrm{~K})$ the dislocations are annihilated as indicated by the decrease of the dislocation density.

In wet atmosphere the diamond is graphitized on the sur- face of the voids between the neighboring particles. The formation of graphite on the surface around the contact points lowers the shear stresses responsible for the dislocation production by increasing the contact surface between the diamond particles. The shear stresses are also decreased due to hydrostatic pressure exerted by the compressed graphite present in the voids between diamond crystals. To the contrary, in samples that did not graphitize, these voids are empty and large shear stresses exist at all temperatures. These effects are responsible for the lower dislocation density in the graphitized samples.

After the raw powder was compressed at $1073 \mathrm{~K}$ the average crystal size was still larger than 1 micron (this is around the upper limit of the crystal size measurable by the $\mathrm{x}$-ray technique). When temperature was increased to above $1073 \mathrm{~K}$, the average crystallite sizes were reduced to the submicron range. In the case of graphite-free diamond, this decrease continued up to $1470 \mathrm{~K}$, when the average crystallite sizes reached $46 \mathrm{~nm}$. Later this process was reversed and at $1760 \mathrm{~K}$ crystallite sizes increased to $75 \mathrm{~nm}$. This effect together with the decrease of the dislocation density is probably associated with polygonization of deformed crystals resulted due to the rearrangement of the dislocation structure into a lower energy configuration. This mechanism has been outlined by Ziman. ${ }^{13}$

\section{CONCLUSION}

The influence of graphitization on strain relaxation in diamond was investigated by evaluating the x-ray diffraction peak profiles using the Fourier coefficients of ab initio theoretical sizes and strain profiles. The procedure allows determining the mean crystallite size, the distribution of sizes, and the density as well as the character of the dislocations induced in diamond crystals after HPHT treatment. The method was applied to two different sets of samples. In one case the graphitization of diamond was accelerated by the presence of water catalyst, in another, graphitization of diamond was practically nonexistent.

The size of diamond crystallites is reduced significantly, by three orders of magnitude, by compaction at temperatures higher than $1070 \mathrm{~K}$. Responsible for this effect is the dislocations formation in the bulk diamond micron size crystals. At the maximum temperature of $1760 \mathrm{~K}$ used in our experiments a strain relaxation by annealing is observed in notgraphitized diamond.

Different dislocation densities were obtained for different treatment procedures. It was found that for the significantly graphitized diamond crystals the dislocation density was by a factor of 2 smaller then for the crystals in which the graphitization was prevented. Screw dislocations, which were practically absent in the initial powder, became abundant after high pressure treatment at $1270 \mathrm{~K}$. At increased temperatures, in graphitized samples the relative concentration of screw dislocations remained smaller than for not-graphitized samples. We interpret these observations as a result of hydrostatic conditions formed in the sample after graphite filled up the voids between diamond crystals. 


\section{ACKNOWLEDGMENTS}

This work was supported by the Hungarian Scientific Research Fund, OTKA, Grant Nos. T031786, T034666, and T029701 and the U.S. Department of Energy, Office of In- dustrial Technology program: Advanced Materials for $\mathrm{Fu}-$ ture. J.G. is grateful for the financial support of Magyary Zoltán postdoctoral program of Foundation for Hungarian Higher Education and Research (AMFK).
${ }^{1}$ F. P. Bundy, J. Chem. Phys. 38, 631 (1963).

${ }^{2}$ J. Qian, C. Pantea, G. A. Voronin, and T. W. Zerda, J. Appl. Phys. 90, 1632 (2001).

${ }^{3}$ T. Evans, in The Properties of Diamonds, edited by J. E. Field (Academic, London, 1979), pp. 403-424.

${ }^{4}$ C. Pantea, J. Qian, G. A. Voronin, and T. W. Zerda, J. Appl. Phys. 91, 1957 (2002).

${ }^{5}$ J. B. Parise, D. J. Weidner, J. Chen, R. C. Liebermann, and G. Chen, Annu. Rev. Mater. Sci. 28, 349 (1998).

${ }^{6}$ T. Ungár, J. Gubicza, G. Ribárik, and A. Borbély, J. Appl. Crystallogr. 34, 298 (2001).

${ }^{7}$ G. Ribárik, T. Ungár, and J. Gubicza, J. Appl. Crystallogr. 34, 669 (2001).
${ }^{8} \mathrm{M}$. Wilkens, in Fundamental Aspects of Dislocation Theory, conference proceedings, National Bureau of Standards, Washington, D.C., 1969, edited by J. A. Simmons, R. de it, and R. Bullough (U.S. National Bureau of Standards, Washington, D.C., 1970), Vol. 2, pp. 1195-1221.

${ }^{9}$ T. Ungár and A. Borbély, Appl. Phys. Lett. 69, 3173 (1996).

${ }^{10}$ T. Ungár and G. Tichy, Phys. Status Solidi A 171, 425 (1999).

${ }^{11}$ H. J. McSkimin and P. Andreatch, J. Appl. Phys. 43, 2944 (1972).

${ }^{12}$ T. Ungár, I. Dragomir, Á. Révész, and A. Borbély, J. Appl. Crystallogr. 32, 992 (1999).

${ }^{13}$ J. M. Ziman, Models of Disorder (Cambridge University Press, Cambridge, 1979), p. 51. 